\title{
Knockdown of ubiquitin protein ligase E3A affects proliferation and invasion, and induces apoptosis of breast cancer cells through regulation of annexin A2
}

\author{
XIAOFANG ZHOU ${ }^{1,2}$, SHISHAN DENG ${ }^{1,3}$, HAI LIU $^{2}$, YANG LIU $^{2}$, ZHENGWEI YANG $^{3}$, \\ TIANYONG XING ${ }^{4}$, BAOQIAN JING ${ }^{5}$ and XIAOMING ZHANG ${ }^{6}$
}

${ }^{1}$ Department of Anatomy, North Sichuan Medical College; ${ }^{2}$ Department of Otolaryngology,

The Affiliated Hospital of North Sichuan Medical College; ${ }^{3}$ Morphometric Research Laboratory,

North Sichuan Medical College; ${ }^{4}$ Department of General Surgery, The Affiliated Hospital of North Sichuan Medical College; ${ }^{5}$ Institute of Immunology and Molecular Biology, North Sichuan Medical College; ${ }^{6}$ Sichuan Key Laboratory of Medical Imaging, North Sichuan Medical College, Nanchong, Sichuan 637007, P.R. China

Received April 17, 2014; Accepted January 21, 2015

DOI: $10.3892 / \mathrm{mmr} .2015 .3549$

\begin{abstract}
The present study used RNA interference (RNAi) to study how the expression of annexin A2 was affected by ubiquitin protein ligase E3A (UBE3A). In addition, the proliferation, apoptosis and invasiveness of BT-549 breast cancer cells was studied following knockdown of UBE3A. Three pairs of small interfering RNA (siRNA) fragments targeting UBE3A were designed and transfected into the BT-549 cells. The effects of silencing UBE3A were detected by reverse transcription-polymerase chain reaction and western blotting, and the same methods were used to detect the expression levels of annexin A2. Cell proliferation was determined using the Cell Counting kit-8, and flow cytometry and a Transwell chamber assay were used to assess the rate of cell apoptosis and invasion, respectively. Following transfection with the three siRNAs targeting UBE3A for $72 \mathrm{~h}$, the mRNA expression levels of UBE3A were downregulated, as compared with those in the untreated groups, and siRNA1 was the shown to be the most effective siRNA for silencing UBE3A expression. The protein expression levels were concordant with the mRNA expression levels of UBE3A. In addition, the mRNA and protein expression levels of annexin A2 were downregulated. Cellular proliferation and invasion of the siRNA1 group was inhibited as compared with those in the untreated groups, and apoptosis of UBE3A-siRNA1 cells was increased as compared with that in the untreated groups. The results of the present study indicated that UBE3A may regulate the expression of
\end{abstract}

Correspondence to: Dr Shishan Deng, Department of Anatomy, North Sichuan Medical College, 234 Fujiang Road, Nanchong, Sichuan 637007, P.R. China

E-mail: dssgeneral@163.com

Key words: RNA interference, breast cancer, BT-549 cells, proliferation, invasion, apoptosis annexin A2, resulting in promotion of proliferation and invasion and suppression of apoptosis in BT-549 cells.

\section{Introduction}

Ubiquitin protein ligase E3A (UBE3A), also known as human papilloma virus E6-associated protein (E6-AP), is an important member of ubiquitin proteasome systems. The E6/E6-AP complex has previously been shown to target p53 for ubiquitination and degradation through the ubiquitin-proteasome pathway (UPP) in cervical cancer $(1,2)$. It has been reported that abnormal expression of UBE3A is associated with various types of tumor, including prostate, cervical and breast cancer (3-5). However, how the loss of function of UBE3A causes disease pathogenesis remains to be elucidated. A previous study by our group reported that UBE3A was overexpressed in breast cancer and associated with its pathological mechanism (6). Therefore, it may be hypothesized that UBE3A has an important role in breast cancer through its ubiquitin-protein ligase activity.

Previously, the degradation of various proteins has been shown to be mediated by UBE3A, including c-Myc (7), human telomerase reverse transcriptase (8), p27 (9), progesterone receptor-B protein and annexin A1 protein $(10,11)$. To date, these cellular substrates of E6-AP have been identified and characterized; however, the significance of these interactions in disease remains to be fully elucidated.

Annexin A2 is a member of the annexin family, which has been reported to be abundantly expressed in numerous types of malignant tissue, and is believed to have an important role in tumorigenesis and cancer progression (12). A previous study demonstrated that annexin A 2 could be inhibited by RNA interference (RNAi), which resulted in decreased metastasis and invasion of breast cancer cells (13). Furthermore, a previous study by our group showed that annexin A2 was overexpressed and ubiquitinated in breast cancer tissues (14). These findings suggested that annexin A2 may contribute to the pathogenesis of breast cancer, as it is mediated by ubiquitination, and it may be a novel substrate of E6-AP. 
Table I. siRNA sequences.

\begin{tabular}{ll}
\hline Name & \\
\hline siRNA1 & Sequences \\
siRNA2 & Anti-sense strand: 5'-AUGUACAUCCAGUAUACAGTT-3' \\
& Sense strand: 5'-CUCAAAUCAGGAACUGUAUTT-3' \\
siRNA3 & Anti-sense strand: 5'-AUACAGUUCCUGAUUUGAGTT-3' \\
& Sense strand: 5'-GAAGACAAUGCUUUCCAUATT-3' \\
Negative control siRNA & Anti-sense strand: 5'-UAUGGAAAGCAUUGUCUUCTT-3' \\
& Sense strand: 5'-AGGUGACUAGCACUGUUAGTT-3' \\
& Anti-sense strand: 5'-GUAACAGUGCUAGUCACCUTT-3'
\end{tabular}

siRNA, small interfering RNA.

The function of UBE3A in the proliferation, invasion and apoptosis of breast cancer have remained elusive; therefore, the present study used RNAi to silence UBE3A expression in BT-549 breast cancer cells in order to determine its function. The results indicated that UBE3A is involved in carcinogenesis by regulating the levels of annexin A2 in breast cancer cells, which renders it a potential molecular marker of breast cancer and a target of breast cancer therapy.

\section{Materials and methods}

Reagents. The BT-549 human breast cancer cell line was obtained from the Cell Bank of Chinese Academy of Sciences (Beijing, China). RPMI-1640 cell culture medium was purchased from HyClone Laboratories, Inc. (Logan, UT, USA). Lipofectamine ${ }^{\circledR} 2000$ and TRIzol ${ }^{\circledR}$ were obtained from Invitrogen Life Technologies (Carlsbad, CA, USA). Fetal bovine serum (FBS), penicillin and streptomycin were purchased from Gibco Life Technologies (Carlsbad, CA, USA). First Strand cDNA Synthesis kit and the polymerase chain reaction (PCR) kit were purchased from Takara Bio, Inc. (Otsu, Japan). Rabbit monoclonal UBE3A (1:1,000; no. $5571-1)$, rabbit polyclonal $\beta$-actin $(1: 2,000$; no. $5779-1)$ and rabbit polyclonal annexin A2 (1:1,000; no. S0555) primary antibodies, as well as goat anti-rabbit horseradish peroxidase-conjugated immunoglobulin G (1:5,000; no. 3053-1-1) secondary antibody were purchased from Epitomics (Burlingame, CA, USA). Enhanced Chemiluminescence (ECL) Reagent kit, fibronectin and Transwell assay plates were obtained from EMD Millipore (Billerica, MA, USA). Cell Counting Kit-8 (CCK-8) and Annexin V-Fluorescein Isothiocyanate (FITC)/Propidium Iodide (PI) Cell Apoptosis Detection kit were purchased from Nanjing Keygen Biotech., Co., Ltd., Nanjing, China). Negative siRNA and specific UBE3A siRNAs were synthesized by Takara Bio, Inc. Matrigel ${ }^{\circledR}$ was purchased from BD Biosciences (Franklin Lakes, NJ, USA).

Cell culture. The BT-549 human breast cancer cells were cultured in RPMI-1640 supplemented with 10\% FBS, $100 \mathrm{U} / \mathrm{ml}$ penicillin and $100 \mathrm{U} / \mathrm{ml}$ streptomycin at $37^{\circ} \mathrm{C}$ in an atmosphere containing $5 \% \mathrm{CO}_{2}$.
Design and synthesis of siRNA targeting UBE3A. Using siDirect Version 2.0 design software (http://sidirect2.rnai.jp/), three specific siRNAs were designed to target the UBE3A gene, and the negative siRNA fragments were synthesized by Takara Bio, Inc. Specific siRNA sequences are shown in Table I.

Cell transfection. One day prior to transfection, the BT-549 cells were seeded in a six-well plate ( $2 \times 10^{5}$ cells). A total of $5 \mu 1$ siRNA, at $20 \mu \mathrm{M}$ stock, was added to $250 \mu \mathrm{l}$ RPMI-1640. In addition, $5 \mu$ l Lipofectamine ${ }^{\circledR} 2000$ was diluted in the same amount of RPMI-1640 medium. Following an incubation for $5 \mathrm{~min}$ at room temperature, the diluted siRNAs were gently added to the diluted Lipofectamine ${ }^{\circledR} 2000$ medium and incubated for $20 \mathrm{~min}$ at room temperature. Subsequently, the mixture was added to the cells, alongside $1.5 \mathrm{ml}$ serum-free and antibiotic-free RPMI-1640 medium. Following a 6-h incubation, the mixture was removed and $2 \mathrm{ml}$ RPMI-1640 supplemented with 10\% FBS was added to the plate. Cells treated with Lipofectamine ${ }^{\circledR} 2000$ only were considered the control group.

Semi-quantitative reverse transcription $(R T)-P C R$. The BT-549 cells transfected with siRNAs were harvested $72 \mathrm{~h}$ post-transfection. Total RNA was extracted using TRIzol ${ }^{\circledR}$ reagent according to the manufacturer's instructions. The cDNA was synthesized using the PrimeScript RT reagent kit. PCR amplification was conducted using the Taq polymerase enzyme (Takara Bio, Inc.). The PCR reaction program was set as follows: $95^{\circ} \mathrm{C}$ for $1 \mathrm{~min}$, followed by 30 cycles of $94^{\circ} \mathrm{C}$ for $30 \mathrm{sec}, 55^{\circ} \mathrm{C}$ for $30 \mathrm{sec}$ and $72^{\circ} \mathrm{C}$ for $1 \mathrm{~min}$, then a final extension step of $72^{\circ} \mathrm{C}$ for $5 \mathrm{~min}$ and then stored at $4^{\circ} \mathrm{C}$ for further use. $\beta$-actin was used as the internal control. The PCR products were then separated by $1.5 \%$ agarose gel electrophoresis (Takara Bio, Inc.) at $105 \mathrm{~V}$ for $30 \mathrm{~min}$. Images were captured using the GelDoc XR system (Bio-Rad Laboratories, Inc., Hercules, CA, USA), and the images were analyzed using PD QUEST 8.0 software (Bio-Rad Laboratories, Inc.). The sequences of the specific primers are shown in Table II. The experiment was repeated three times.

Western blot analysis. Total protein was extracted from the cells using radioimmunoprecipitation assay buffer (Beyotime Institute of Biotechnology, Haimen, China), $72 \mathrm{~h}$ 
Table II. Polymerase chain reaction primers.

\begin{tabular}{|c|c|c|}
\hline Name & Sequences & Product length (bp) \\
\hline UBE3A & $\begin{array}{l}\text { Forward: 5'-CACTTGTCCGGCTAGAGATGAT-3' } \\
\text { Reverse: 5'-TCCCCATTAGCTTCCTGTAGAC-3' }\end{array}$ & 324 \\
\hline Annexin A2 & $\begin{array}{l}\text { Forward: 5'-TGACGCTGGAGTGAAGAGGAA-3' } \\
\text { Reverse: 5'-GCCCTTAGTGTCTTGCTGGATA-3 }\end{array}$ & 379 \\
\hline$\beta$-actin & $\begin{array}{l}\text { Forward: 5'-GACCCAGATCATGTTTGAGACC-3 } \\
\text { Reverse: 5'-ATCTCCTTCTGCATCCTGTCG-3' }\end{array}$ & 594 \\
\hline
\end{tabular}

UBE3A, ubiquitin protein ligase E3A; bp, base pairs.

post-transfection. Total protein was quantified using the bicinchoninic acid method (Beyotime Institute of Biotechnology). According to the size of the molecular weight of the protein extracts, the appropriate concentration of SDS-PAGE gel (UBE3A, 8\%; annexin A2 and $\beta$-actin, 12\%). Equal amounts of total protein $(30 \mu \mathrm{g})$ were separated by SDS-PAGE. The proteins were then transferred to polyvinylidene fluoride (PVDF) membranes (0.45 $\mu \mathrm{m}$; EMD Millipore) in transfer buffer (Beyotime Institute of Biotechnology) by electroblotting using a Mini-Trans-Blot Electrophoretic Transfer Cell (Bio-Rad Laboratories, Inc.) at $200 \mathrm{~mA}$ for 1-2 h. The PVDF membranes were blocked for $1 \mathrm{~h}$ at room temperature using blocking solution (Beyotime Institute of Biotechnology), and were then incubated with the specified dilutions of the primary antibodies for UBE3A, annexin A2 and $\beta$-actin overnight at $4^{\circ} \mathrm{C}$. The membranes were then washed three times with Tris-buffered saline-Tween (15 $\mathrm{min} /$ wash), prior to an incubation with the recommended dilution of the labeled secondary antibody at $37^{\circ} \mathrm{C}$ for $1 \mathrm{~h}$. The goat anti-rabbit alkaline phosphatase-conjugated antibody was detected colorimetrically using ECL Plus. Images were captured using the GelDoc XR system and were analyzed using Image $\mathrm{Lab}^{\mathrm{TM}} 3.0$ software (Bio-Rad Laboratories, Inc.). $\beta$-actin was used as the internal control. The experiment was repeated three times.

Cell proliferation assay. The CCK-8 assay was performed to examine cell proliferation. The experiment was repeated five times. Cells $\left(2 \times 10^{3} /\right.$ well $)$ in the logarithmic phase were cultured in 96-well culture plates. The cells were divided into the following three groups: siRNA1 group, negative control group and blank control group. The methods of cell transfection were the same as stated above. A total of $10 \mu \mathrm{l} \mathrm{CKK}-8$ reagent was added at four time-points $(24,48,72$ and $96 \mathrm{~h}$ post-transfection) and the plates were incubated at $37^{\circ} \mathrm{C}$ for $2 \mathrm{~h}$. The absorbance was then measured at a wavelength of $450 \mathrm{~nm}$ using a microplate reader (Biochrom Anthos 2010; Autobio Diagnostics Co., Ltd., Zhengzhou, China).

Flow cytometric assay. The Annexin V-FITC/PI Apoptosis Detection kit was used to detect the rate of cell apoptosis according to the manufacturer's instructions. The experiment was repeated five times. Cells $\left(2 \times 10^{5} / \mathrm{ml}\right)$ in the logarithmic phase were cultured in six-well culture plates. The cells were divided into the same three groups as for the cell proliferation assay. Briefly, the cells were collected $72 \mathrm{~h}$ post-transfection, after being washed twice with cold phosphate-buffered saline (PBS). The cells were then suspended in $500 \mu 1 \mathrm{1x}$ binding buffer and subsequently, $5 \mu \mathrm{l}$ Annexin V and PI were added to the cells. The cells were incubated for $15 \mathrm{~min}$ at room temperature in the dark, followed by flow cytometric analysis (FACSCalibur; Becton Dickinson, Franklin Lakes, NJ, USA). The apoptotic rate was calculated as the percentage of Annexin V-positive and PI-negative cells out of the total number of cells. The assay was conducted using a flow cytometer and was analyzed using WinMDI 2.9 software (www.bioon.com).

Cell invasion assay. Transwell chambers were used to perform invasion experiments. Briefly, Matrige ${ }^{\circledR}$ was thawed at $4^{\circ} \mathrm{C}$ overnight, and was then diluted with an equal amount of PBS. A total of $80 \mu \mathrm{l}$ diluted Matrige ${ }^{\circledR}$ was added to the upper chamber, and $30 \mu \mathrm{l}$ fibronectin was added to the lower chamber of a 24-well Transwell plate (24-well insert; pore size, $8 \mu \mathrm{m}$; EMD Millipore). The Transwell chamber was then incubated at $37^{\circ} \mathrm{C}$ for $\geq 4 \mathrm{~h}$. The cells were harvested from the cell culture plates $72 \mathrm{~h}$ post-transfection, and were washed three times with RPMI-1640 supplemented with $1 \%$ FBS. The cells $\left(4 \times 10^{4}\right.$ cells) were seeded into the upper chamber, and $600 \mu 1$ RPMI-1640 containing adhesive stubstrate (diluted Matrigel ${ }^{\circledR}$ ) was added to the lower chamber. The Transwell chamber was incubated at $37^{\circ} \mathrm{C}$ for $24 \mathrm{~h}$. The cells that did not invade through the pores were removed using a cotton swab, whereas the cells on the lower surface of the membrane were stained with crystal violet (Beyotime Institute of Biotechnology) and counted using an IX53-F32PH microscope (Olympus Corp., Tokyo, Japan). The experiment was repeated tree times.

Statistical analysis. The results are presented as the mean \pm standard deviation. Statistical analyses were performed by one-way analysis of variance using SPSS version 13.0 software (SPSS Inc., Chicago, IL, USA). $\mathrm{P}<0.05$ was considered to indicate a statistically significant difference.

\section{Results}

mRNA expression levels of UBE3A and annexin A2 in BT-549 cells. The relative mRNA expression levels of UBE3A and annexin $\mathrm{A} 2$ were calculated as UBU3A/ $\beta$-actin and annexin A2/ $\beta$-actin, respectively. The UBE3A specific siRNAs 
Table III. Relative mRNA expression levels of UBE3A and annexin A2 in BT-549 human breast cancer cells.

\begin{tabular}{lccc}
\hline Group & $\mathrm{n}$ & UBE3A/ $\beta$-actin & Annexin A2/ $\beta$-actin \\
\hline siRNA1 group & 3 & $0.15 \pm 0.016$ & $0.942 \pm 0.032$ \\
siRNA2 group & 3 & $0.32 \pm 0.026$ & $1.009 \pm 0.014$ \\
siRNA3 group & 3 & $0.23 \pm 0.016$ & $0.99 \pm 0.02$ \\
Negative siRNA group & 3 & $0.59 \pm 0.014$ & $1.333 \pm 0.022$ \\
Lipofectamine 2000 group & 3 & $0.587 \pm 0.021$ & $1.326 \pm 0.018$ \\
Control group & 3 & $0.592 \pm 0.027$ & $1.336 \pm 0.026$ \\
F & & 285.197 & 219.939 \\
P-value & & $\leq 0.05$ & $\leq 0.05$ \\
\hline
\end{tabular}

siRNA, small interfering RNA; UBE3A, ubiquitin protein ligase E3A; n, number of experiments.

A

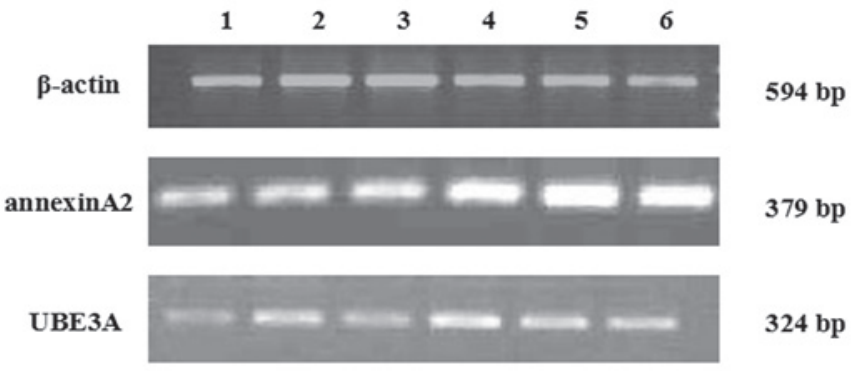

B

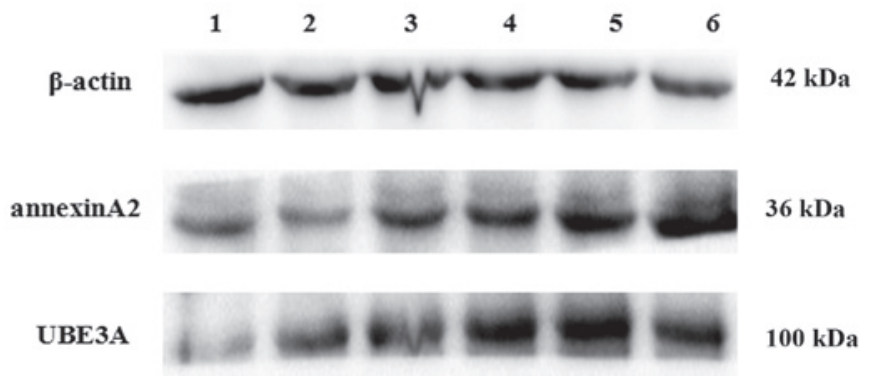

Figure 1. BT-549 human breast cancer cells were transfected with siRNA1, siRNA2, siRNA3 and negative control siRNA, using Lipofectamine ${ }^{\circledR} 2000$. The cells treated with Lipofectamine ${ }^{\circledR} 2000$ only were considered the control. Lane 1, siRNA1 group; lane 2, siRNA2 group; lane 3, siRNA3 group; lane 4, negative control group; lane 5, Lipofectamine ${ }^{\circledR} 2000$ group; lane 6, blank control group. (A) Total RNA was extracted from the cells $72 \mathrm{~h}$ post-transfection. Reverse transcription polymerase chain reaction was performed to measure the relative mRNA expression levels of UBE3A and annexin A2. The PCR products were seperated by $1.5 \%$ agarose gel electrophoresis. The mRNA expression levels of $\beta$-actin served as a loading and normalization control. (B) Protein expression levels of UBE3A and annexin A2 protein expression levels were determined by western blotting. The protein expression levels of $\beta$-actin served as a loading and normalization control. Data were averaged from three independent experiments. siRNA, small interfering RNA; UBE3A, ubiquitin protein ligase E3A.

effectively silenced the mRNA expression levels of UBE3A, as compared with levels in the control groups $(n=3, P \leq 0.01)$. In addition, following UBE3A knockdown with siRNA1, the relative mRNA expression levels of annexin A2 were downregulated, as compared with those in the control groups $(n=3$, $\mathrm{P} \leq 0.05$ ) (Fig. 1A and Table III).

Protein expression levels of UBE3A and annexin A2 in BT-549 cells. The relative protein expression levels of UBE3A and annexin A2 were calculated as UBU3A/ $\beta$-actin and annexin $A 2 / \beta$-actin, respectively. The protein expression levels were concordant with the mRNA expression levels of UBE3A and annexin A2. siRNA1 was shown to be the most effective siRNA for silencing UBE3A mRNA as well as protein expression; therefore, the siRNA1 group was selected as the experimental group for subsequent assays (Fig. 1B and Table IV).

Cell proliferation. Proliferation of the BT-549 cells in the siRNA1 group was significantly inhibited 24, 48, 72 and $96 \mathrm{~h}$ post-transfection, as compared with that of the blank control group $(\mathrm{n}=5, \mathrm{P}<0.01)$. However, there were no statistically significant differences detected between the negative siRNA group and the blank control group at the four post-transfection time-points ( $\mathrm{n}=5, \mathrm{P}>0.05)$ (Fig. 2 and Table V).

Cell apoptosis. The rate of apoptosis of the cells in the siRNA1 group, negative control group and blank control group 
Table IV. Relative protein expression levels of UBE3A and annexin A2 in BT-549 human breast cancer cells.

\begin{tabular}{lccc}
\hline Groups & $\mathrm{n}$ & UBE3A/ $\beta$-actin & Annexin A2/ $\beta$-actin \\
\hline siRNA1 group & 3 & $0.276 \pm 0.019$ & $0.452 \pm 0.033$ \\
siRNA2 group & 3 & $0.332 \pm 0.025$ & $0.547 \pm 0.036$ \\
siRNA3 group & 3 & $0.312 \pm 0.022$ & $0.543 \pm 0.048$ \\
Negative siRNA group & 3 & $0.471 \pm 0.031$ & $0.833 \pm 0.03$ \\
Lipofectamine 2000 group & 3 & $0.507 \pm 0.021$ & $0.846 \pm 0.041$ \\
Control group & 3 & $0.478 \pm 0.031$ & $0.887 \pm 0.027$ \\
F & & 47.655 & 81.604 \\
P-value & & $\leq 0.05$ & $\leq 0.05$ \\
\hline
\end{tabular}

siRNA, small interfering RNA; UBE3A, ubiquitin protein ligase E3A; n, number of experiments.

Table V. Absorbance of BT-549 human breast cancer cells at various time-points.

\begin{tabular}{lccrr}
\hline Groups & $24 \mathrm{~h}$ & $48 \mathrm{~h}$ & $72 \mathrm{~h}$ & $96 \mathrm{~h}$ \\
\hline siRNA1 & $0.5244 \pm 0.0130^{\mathrm{a}}$ & $0.5528 \pm 0.0147$ & $0.523 \pm 0.0120$ & $0.695 \pm 0.0190$ \\
Negative siRNA & $0.6218 \pm 0.0110^{\mathrm{b}}$ & $0.6744 \pm 0.0143$ & $0.7432 \pm 0.0184$ & $0.9318 \pm 0.0094$ \\
Control & $0.6668 \pm 0.0163$ & $0.7098 \pm 0.0143$ & $0.796 \pm 0.0268$ & $0.9622 \pm 0.02$
\end{tabular}

siRNA, small interfering RNA. $\mathrm{n}=5$; ${ }^{\mathrm{a}} \mathrm{P}<0.05$, siRNA1 group vs. control group; ${ }^{\mathrm{b}} \mathrm{P}>0.05$ negative siRNA group vs. control group.

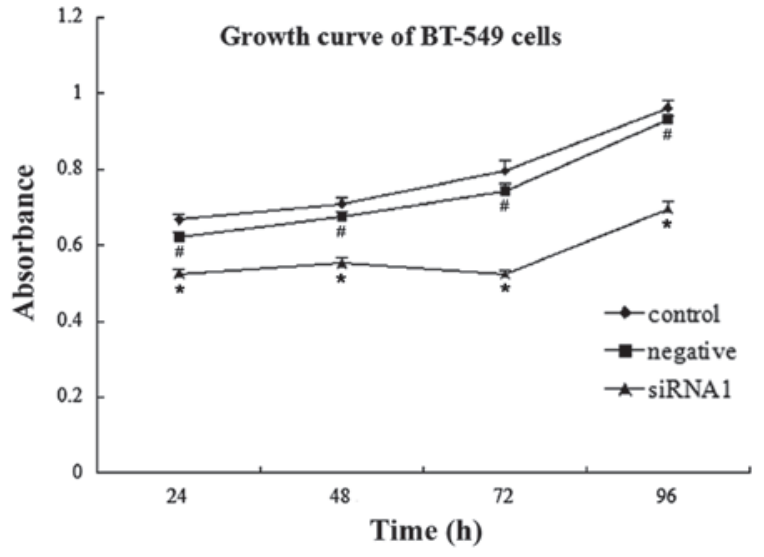

Figure 2. Cell proliferation curves of the UBE3A-siRNA1 group, negative control group and blank control group. BT-549 human breast cancer cells were incubated for the indicated time-periods $(24,48,72$ and $96 \mathrm{~h})$. As a measurement of cell viability, the relative absorbance of the cells was obtained using the Cell Counting kit- $8(n=5)$. Values are presented as the mean \pm standard deviation. ${ }^{*} \mathrm{P}<0.05$, as compared with the negative siRNA and blank contro groups; ${ }^{~} \mathrm{P} \geq 0.05$, negative control group as compared with the blank control group. siRNA, small interfering RNA; UBE3A, ubiquitin protein ligase E3A.

was $11.104 \pm 1.1935 \%, 2.852 \pm 0.7213 \%$ and $2.788 \pm 0.3954 \%$, respectively, $72 \mathrm{~h}$ post-transfection. The rate of apoptosis was significantly higher in the siRNA1 group, as compared with the control groups $(\mathrm{n}=5, \mathrm{P}<0.05)$ (Fig. 3).

Cell invasion. The number of invasive cells in the siRNA1 group, negative control group and blank control group were $53.96 \pm 4.55,93.16 \pm 3.91$ and $94.43 \pm 3.71$, respectively, $72 \mathrm{~h}$ post-transfection. The number of invasive cells in the siRNA group was significantly lower as compared with that in the blank control group $(\mathrm{n}=10, \mathrm{P}<0.01)$ (Fig. 4).

\section{Discussion}

The UPP is an important pathway of protein degradation for numerous proteins vital to cellular regulation and function. Furthermore, the ubiquitin-proteasome system is a crucial regulator of the cell cycle, and it is well-known that abnormal cell cycle control may lead to oncogenesis (15-17). A previous study demonstrated that UPP dysregulation has an important role in mammary tumorigenesis (18). UBE3A is an important member of the ubiquitin-ligase family and is one of the key enzymes associated with the maintenance of normal cellular physiological functions (15). Abnormal alterations of UBE3A may cause the development of various diseases.

RNAi has recently been identified as being capable of gene silencing. RNAi is mediated by double-stranded RNA, which can degrade specific target mRNAs (4). The present study used chemically synthesized siRNAs to suppress the expression of UBE3A. Chemical synthesis of siRNA has previously been shown to effectively block gene expression (19). According to the principle of RNAi, specific siRNAs were synthesized in vitro, and were then transfected into BT-549 human breast cancer cells. The interference effects were detected using semi-quantitative RT-PCR and western blotting. The results of the present study demonstrated that three specific siRNAs were able to effectively inhibit the expression of UBE3A. siRNA1 was shown to exhibit the best interference effect. Following effective UBE3A gene knockdown, the expression levels of annexin A2 
A

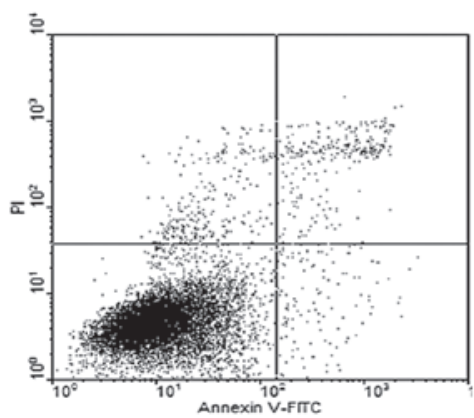

B

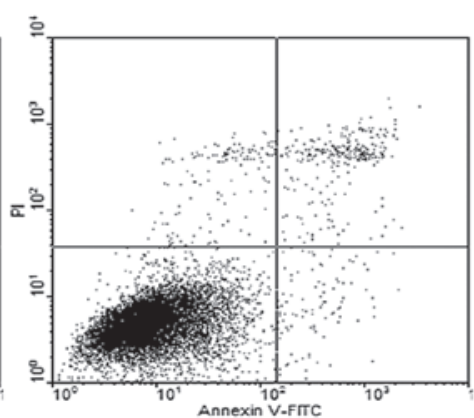

C

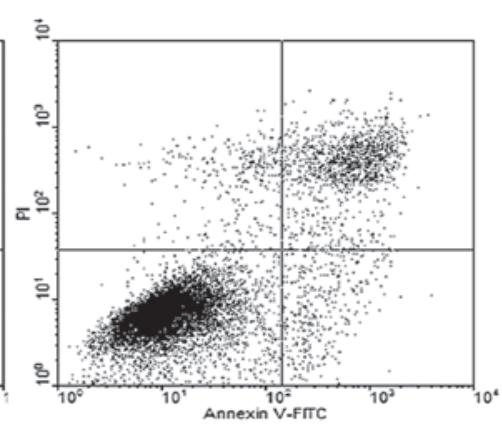

Figure 3. Percentage of apoptotic cells in each group was detected by flow cytometry and analyzed using WinMDI 2.9 software. The cells were harvested and stained with Annexin V-FITC and PI for cell apoptosis analysis, and the data from three independent experiments were averaged. (A) Blank control group, (B) negative control group and (C) UBE3A-siRNA1 group. FITC, fluorescein isothiocyanate; PI, propidium iodide; siRNA, small interfering RNA; UBE3, ubiquitin protein ligase E3A.
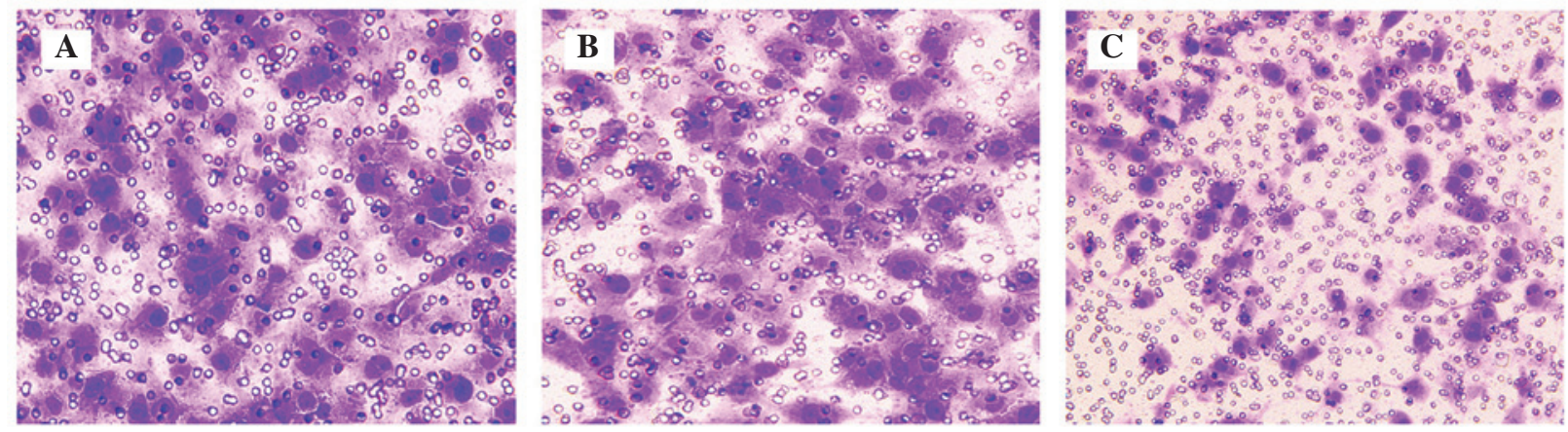

Figure 4. Cells that invaded through the pores of a membrane to the lower chamber of a Transwell plate were stained with crystal violet solution. Quantification of the invasion is expressed as the number of invading cells per high-power field (magnification, x20). (A) Blank control group, (B) negative control group and (C) UBE3A-siRNA1 group. siRNA, small interfering RNA; UBE3, ubiquitin protein ligase E3A.

were downregulated. This may have an adverse effect on certain proteins that are degraded in an E6-AP-dependent manner. Shimoji et al (10) previously reported that RNAi-mediated downregulation of endogenous E6-AP increased the levels of endogenous annexin A1 protein, but had no effect on the accumulation of endogenous annexin A2 protein.

The present study aimed to determine why the expression of annexin A2 was downregulated following knockdown of UBE3A expression. The results suggested that UBE3A may have a role in controlling the functions of annexin A2 in breast cancer cells. It may be hypothesized that ubiquitinated annexin A2 was not degraded, but its function, activity or position may have been altered, which was regulated by UBE3A in BT-549 breast cancer cells. Another hypothesis may be that following knockdown of UBE3A, annexin A2 may be degraded via UPP, or another pathway. Previous studies have suggested that polyubiquitinated protein may be generally degraded by the $26 \mathrm{~S}$ proteasome; however, if polyubiquitinated proteins are not connected to the target protein 48 or 63-bit lysines, but to the $6,11,27,29$ and 33 lysines, the ubiquitinated proteins may not be degraded, but their funtions may be altered $(20,21)$. Furthermore, monoubiquitinated proteins may not be degraded; however, their activity, positioning or structure may be modified, thus regulating the endocytosis pathway, histone modification, gene transcription and nuclear protein localization (22). Future studies may detect the ubiquitinated position or numbers of ubiquitinated annexin A2 in the BT-549 breast cancer cells prior to as well as following UBE3A RNAi, in order to further study the association between annexin A2 and UBE3A. Exploring the role of UBE3A in the transcriptional regulation of annexin $\mathrm{A} 2$ or other genes may be an interesting area of research.

Treatment and prognosis of breast cancer are complex and are associated with numerous factors in vivo and in vitro. Cell proliferation, apoptosis and invasion are considered the most important factors affecting the treatment and prognosis of breast cancer. In the present study, a CCK- 8 assay, flow cytometry and a Transwell assay were used to detect the proliferation, apoptosis and invasion of BT-549 cells following silencing of UBE3A. The results demonstrated that the proliferation of BT-549 cells was restrained following transfection withUBE3A siRNA. The rate of apoptosis of the cells in the siRNA1 group was increased, and the invasiveness of the cells was decreased. All of these differences were statistically significant when comparing the siRNA1 group with the control groups.

The present study aimed to determine how UBE3A could influence proliferation, apoptosis and invasion of breast cancer cells. A previous study demonstrated that UBE3A was able to impact the function of cells, including transcription, signal transduction, cell survival, cell cycle control and DNA repair (23). In addition, it has been reported that UBE3A can promote proliferation and inhibit apoptosis of cervical cancer cells (4). In prostate cancer cells, UBE3A has been shown to regulate the phosphoinositide 3-kinase-Akt pathway, which 
results in increased prostate cell growth, proliferation and decreased apoptosis (24). Therefore, it was hypothesized that in breast cancer cells, UBE3A may affect cell signaling pathways and influence cell biological behavior.

The present study also demonstrated that following knockdown of UBE3A, the expression of annexin A2 was downregulated. Annexin A2 has previously been demonstrated to promote the development, invasion and metastasis of breast cancer (13), which may explain the simultaneous influence on proliferation, apoptosis and invasion of breast cancer cells. Furthermore, UBE3A may regulate other cancer-associated proteins, which have important roles in the pathogenesis of breast cancer and directly or indirectly lead to cancer through regulating cell proliferation, migration, invasion and apoptosis. E6-AP has also been reported to promote the degradation of p53 $(3,25,26)$. Altered degradation of p53 may also affect cell cycle progression and induce apoptosis. A future aim of our group is to use two-dimensional polyacrylamide gel electrophoresis and matrix-assisted laser desorption/ionization tandem time-of-flight mass spectrometry incorporated with online database research to identify the effect of knockdown of UBE3A expression on the levels of various proteins.

In conclusion, the results of the present study indicated that UBE3A has a role in regulating the functions of annexin A2 and a positive role in the development of breast cancer. These results suggested that UBE3A may be a potential marker for treatment of breast cancer. However, the specific mechanisms underlying the functions of UBE3A in breast cancer require further research.

\section{Acknowledgements}

The present study was supported by grants from the Natural Science Foundation of China (grant no. 81172496), the Sichuan Science and Technology Support Program (grant no. 2009SZ0116) and the Sichuan Department of Education (grant no. 10ZA075).

\section{References}

1. Yamamoto Y, Huibregtse JM and Howley PM: The human E6-AP gene (UBE3A) encodes three potential protein isoforms generated by differential splicing. Genomics 41: 263-266, 1997.

2. Scheffner M, HuibregtseJM, Vierstra RD, and Howley PM: The HPV-16 E6 and E6-AP complex functions as a ubiquitin-protein ligase in the ubiquitination of p53. Cell 75: 495-505, 1993.

3. Khan OY, Fu G, Ismail A, et al: Multifunction steroid receptor coactivator, E6-associated protein, E6-AP is involved in development of the prostate gland. Mol Endocrinol 20: 544-559, 2006.

4. Xiu XX, Zhang SL, Lu XY, Liang MY, Yu J and Hou JP: SiRNA inhibition of E6AP expression in cervical cancer cells. Zhonghua Bing Li Xue Za Zhi 37: 822-825, 2008 (In Chinese).

5. Ramamoorthy S, Tufail R, Hokayem JE, et al: Overexpression of ligase defective E6-associated protein, E6-AP, results in mammary tumorigenesis. Breast Cancer Res Treat 132: 97-108, 2012.
6. Deng S, Zhou H, Xiong R, et al: Over-expression of genes and proteins of ubiquitin specific peptidases (USPs) and proteasome subunits (PSs) in breast cancer tissue observed by the methods of RFDD-PCR and proteomics. Breast Cancer Res Treat 104: 21-30, 2007.

7. Liu X, Disbrow GL, Yuan H, Tomaic V, and Schlegel R: Myc and human papillomavirus type $16 \mathrm{E} 7$ genes cooperate to immortalize human keratinocytes. J Virol 81: 12689-12695, 2007.

8. Liu X, Yuan H, Fu B, et al: The E6AP ubiquitin ligase is required for transactivation of the hTERT promoter by the human papillomavirus E6 oncoprotein. J Biol Chem 280: 10807-10816, 2005.

9. Mishra A, Godavarthi SK and Jana NR: UBE3A/E6-AP regulates cell proliferation by promoting proteasomal degradation of $\mathrm{p} 27$. Neurobiol Dis 36: 26-34, 2009.

10. Shimoji T, Murakami K, Sugiyama Y, et al: Identification of annexin A1 as a novel substrate for E6AP-mediated ubiquitylation. J Cell Biochem 106: 1123-1135, 2009.

11. Ramamoorthy S, Dhananjayan SC, Demayo FJ and Nawaz Z: Isoform-specific degradation of PR-B by E6-AP is critical for normal mammary gland development. Mol Endocrinol 24: 2099-2113, 2010.

12. Lokman NA, Ween MP, Oehler MK, and Ricciardelli C: The role of annexin A2 in tumorigenesis and cancer progression. Cancer Microenviron 4: 199-208, 2011.

13. Sharma MR, Koltowski L, Ownbey RT, Tuszynski G and Sharma MC: Angiogenesis-associated protein annexin II in breast cancer: selective expression in invasive breast cancer and contribution to tumor invasion and progression. Exp Mol Pathol 81: 146-156, 2006.

14. Deng S, Jing B, Xing T, Hou L and Yang Z: Overexpression of annexin A2 is associated with abnormal ubiquitination in breast cancer. Genomics Proteomics Bioinformatics 10: 153-157, 2012.

15. Ramamoorthy S and Nawaz Z: E6-associated protein (E6-AP) is a dual function coactivator of steroid hormone receptors. Nucl Recept Signal 6: e006, 2008.

16. Yamasaki L and Pagano M: Cell cycle, proteolysis and cancer. Curr Opin Cell Biol 16: 623-628, 2004.

17. Micel LN, Tentler JJ, Smith PG and Eckhardt GS: Role of ubiquitin ligases and the proteasome in oncogenesis: novel targets for anticancer therapies. J Clin Oncol 31: 1231-1238, 2013.

18. Dees EC and Orlowski RZ: Targeting the ubiquitin-proteasome pathway in breast cancer therapy. Future Oncol 2: 121-135, 2006.

19. Spänkuch-Schmitt B, Bereiter-Hahn J, Kaufmann M and Stredhardt K: Effect of RNA sileneing of polo-like kinase-1 (PLK1) on apoptosis and spindle formation in human cancer cells. J Natl Cancer Inst 94: 1863-1877, 2002.

20. Liu S and Chen ZJ: Expanding role of ubiquitination in NF- $\kappa B$ signaling. Cell Res 21: 6-21, 2011.

21. Adhikari A and Chen ZJ: Diversity of polyubiquitin chains. Dev Cell 16: 485-486, 2009.

22. Sadowski M, Suryadinata R, Tan AR, Roesley SN and Sarcevic B: Protein monoubiquitination and polyubiquitination generate structural diversity to control distinct biological processes. IUBMB Life 64: 136-142, 2012.

23. Lochab S, Pal P, Kanaujiya JK, et al: Proteomic identification of E6AP as a molecular target of tamoxifen in MCF7 cells. Proteomics 12: 1363-1377, 2012.

24. Srinivasan $\mathrm{S}$ and Nawaz Z: E3 ubiquitin protein ligase, E6-associated protein (E6-AP) regulates PI3K-Akt signaling and prostate cell growth. Biochim Biophys Acta 1809: 119-127, 2011.

25. Jiang YH, Armstrong D, Albrecht U, et al: Mutation of the Angelman ubiquitin ligase in mice causes increased cytoplasmic p53 and deficits of contextual learning and long-term potentiation. Neuron 21: 799-811, 1998.

26. Mishra A and Jana NR: Regulation of turnover of tumor suppressor $\mathrm{p} 53$ and cell growth by E6-AP, a ubiquitin protein ligase mutated in Angelman mental retardation syndrome. Cell Mol Life Sci 65: 656-666, 2008. 